\title{
Nuclease-mediated depletion biases in ribosome footprint profiling libraries
}

\author{
BORIS ZINSHTEYN, ${ }^{1,2}$ JAMIE R. WANGEN, ${ }^{1,2}$ BOYANG HUA, ${ }^{1,2}$ and RACHEL GREEN ${ }^{1,2}$ \\ ${ }^{1}$ Howard Hughes Medical Institute (HHMI) \\ ${ }^{2}$ Department of Molecular Biology and Genetics, Johns Hopkins University School of Medicine, Baltimore, Maryland 21205, USA
}

\begin{abstract}
Ribosome footprint profiling is a high-throughput sequencing-based technique that provides detailed and global views of translation in living cells. An essential part of this technology is removal of unwanted, normally very abundant, ribosomal RNA sequences that dominate libraries and increase sequencing costs. The most effective commercial solution (Ribo-Zero) has been discontinued as a standalone product and a number of new, experimentally distinct commercial applications have emerged on the market. Here we evaluated several commercially available alternatives designed for RNA-seq of human samples and find them generally unsuitable for ribosome footprint profiling. We instead recommend the use of customdesigned biotinylated oligos, which were widely used in early ribosome profiling studies. Importantly, we warn that depletion solutions based on targeted nuclease cleavage significantly perturb the high-resolution information that can be derived from the data, and thus do not recommend their use for any applications that require precise determination of the ends of RNA fragments.
\end{abstract}

Keywords: RNA-seq; Ribo-Zero; rRNA depletion; ribo-seq; ribosome profiling

\section{INTRODUCTION}

Ribosome footprint profiling (Ingolia et al. 2009, 2019; McGlincy and Ingolia 2017) provides nucleotide-precision snapshots of ribosome positions transcriptome-wide. This technique has been used by many groups to study a wide variety of biological problems, ranging from the mechanisms of translation regulation and mRNA quality control to questions about viral infection, stem cell differentiation, and mouse neurobiology. The technique works by sequencing the short ( $\sim 30 \mathrm{nt})$ ribosome-protected mRNA fragments (footprints) that result from nuclease digestion of a cellular lysate (Steitz 1969; Kozak and Shatkin 1976; Wolin and Walter 1988). This nuclease digestion process inevitably causes widespread nicks in the ribosomal RNA (rRNA), producing an abundance of short rRNA fragments that copurify with intact ribosomes and comigrate with the ribosome footprints on polyacrylamide gels. Thus, the resulting sequencing data sets largely consist of rRNA sequences (Ingolia et al. 2009), limiting the sequencing depth of useful ribosome footprints.

Some early studies were able to mitigate contamination with rRNA fragments by gel purifying a very tight distribution of $\sim 30$ nt RNA fragments (the expected size of a ribosome footprint) to avoid prominent rRNA fragments (Guo

Corresponding author: borisz@jhmi.edu

Article is online at http://www.rnajournal.org/cgi/doi/10.1261/rna. 075523.120. et al. 2010), an approach which will not work for all species and samples. Cutting a narrow range of fragments also excludes other lengths of ribosome footprints, which report on the often substantial populations of ribosomes lacking A-site tRNAs (Lareau et al. 2014; Wu et al. 2019), trapped on truncated mRNAs (Guydosh and Green 2014; Arribere and Fire 2018; D'Orazio et al. 2019), or engaged in collisions (Guydosh and Green 2014).

Later studies used subtractive hybridization with biotinylated oligos to deplete the most abundant rRNA fragments (Brar et al. 2012; Ingolia et al. 2012), but variation in sample and library preparation can lead to differences in the number and identity of the most abundant contaminants, often requiring design of new probes with each modification of protocol. Commercial rRNA depletion solutions for RNAseq are generally designed to work on intact or lightly fragmented rRNA, whose fragments are present in equimolar ratios. These assumptions do not apply to the rRNA fragments in ribosome profiling libraries, which are hugely biased by nuclease digestion and size selection. The standard rRNA depletion reagent for ribosome profiling became Ribo-Zero, which consisted of internally

(C) 2020 Zinshteyn et al. This article is distributed exclusively by the RNA Society for the first 12 months after the full-issue publication date (see http://rnajournal.cshlp.org/site/misc/terms.xhtml). After 12 months, it is available under a Creative Commons License (Attribution-NonCommercial 4.0 International), as described at http://creativecommons. org/licenses/by-nc/4.0/. 
biotinylated and fragmented RNA antisense to the entire rRNA (Sooknanan 2009) and was able to deplete a broad range of fragments from ribosome profiling libraries, albeit with varying success (McGlincy and Ingolia 2017).

The recent discontinuation of RiboZero as a standalone product (though currently available for Human/Mouse/ Rat as a component of some RNA-seq kits) led us to test several alternative commercial solutions designed for rRNA depletion of human samples. We found that the commercial products that we tested were at best only modestly effective at depleting rRNA from ribosome profiling libraries. More importantly, we found that methods utilizing targeted nucleolytic degradation of rRNA, such as by $\mathrm{RNaseH}$, caused off-target trimming and degradation of ribosome footprints. Broadly speaking, these treatments reduce the fraction of mappable sequences, perturb global gene expression measurements and blur positional information.

\section{RESULTS}

In order to identify an alternative rRNA depletion option for ribosome footprint profiling we tested a small number of commercial depletion technologies designed for RNA-seq of human samples, alongside the remainder of our existing Ribo-Zero stock (hereafter referred to as legacy Ribo-Zero). In brief, our protocol (McGlincy and Ingolia 2017; Wu et al. 2019) consists of RNasel digestion of cellular lysates, followed by pelleting ribosomes over a sucrose cushion and isolation of fragments by PAGE. To prepare them for sequencing (Fig. 1A), these fragments are then ligated to a $3^{\prime}$ preadenylated DNA linker, depleted of rRNA fragments, reverse transcribed, circularized, and PCR amplified for Illumina sequencing. For this comparison, we prepared ribosome footprints from the same HEK293T cell lysate in duplicate, cutting broadly to isolate 15 to 40 nt fragments, which include the standard $30 \mathrm{nt}$ ribosome footprints, as well as the $\sim 15-18$ and 21 nt fragments that report on mRNA cleavage (Guydosh and Green 2014) and unoccupied A sites (Wu et al. 2019), respectively. We split these
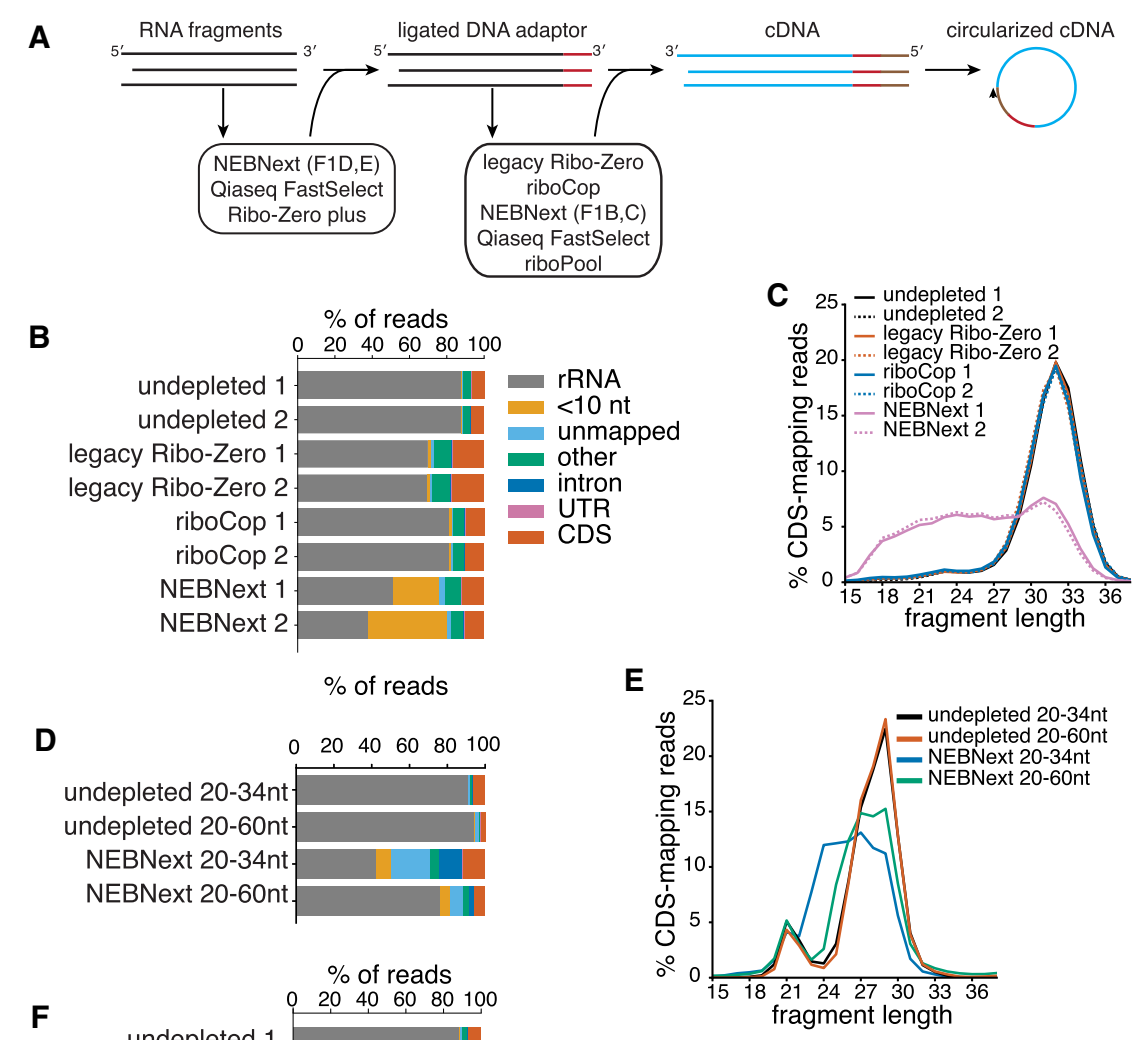

FIGURE 1. rRNA depletion and fragment length perturbation by commercial kits. (A) Simplified flowchart of library preparation for ribosome profiling, with steps at which various rRNA depletion strategies were performed indicated. Arrowhead on circularized cDNA indicates footprint $5^{\prime}$ end. (B) Fractions of reads that are too short, unmappable, or mapping to various genomic regions for different rRNA depletion methods in HEK293T cells. (C) Length distribution of ribosome footprints mapping to coding regions. $(D, E)$ Same as $B$ and $C$ for two different ranges of fragment size selection, depleted with the NEB kit in K562 cells. $(F, G)$ Same as $B$ and $C$, for various depletions strategies in K562 cells. In panel $G$, only RiboZero plus data sets are shown, as others did not have changes in length distribution.

each into four identical groups after $3^{\prime}$ linker ligation (Fig. 1A), to be depleted with Ribo-Zero (Illumina), RiboCop (Lexogen), NEBNext (NEB), or left undepleted. RiboCop is an affinity depletion method that uses complementary biotinylated DNA oligos targeting the rRNA. The NEBNext rRNA depletion kit uses antisense DNA oligos that tile the entire rRNA to target RNaseH-mediated degradation of rRNA. As described above, Ribo-Zero consists of biotinylated rRNA fragments complementary to entire rRNAs (Sooknanan 2009). 
After sequencing the resulting libraries, we found that the undepleted libraries contained $6.6 \pm 0.13 \%$ of reads mapping to coding regions (CDS) (Fig. 1B; Supplemental Table S1). Legacy Ribo-Zero increased this to $16.6 \pm$ $0.33 \%$, while RiboCop and NEBNext yielded $9.78 \pm$ $0.12 \%$ and $10.79 \pm 0.95 \%$, respectively. While all of these approaches increased the number off useable reads, we noticed that the NEBNext kit produced a large fraction of short and unmappable fragments ("<10 nt" and "unmapped" in Fig. 1B). To investigate this further, we looked at the fragment size distribution in these libraries (Fig. 1C). Ribosome footprint profiling in human cells normally produces fragments of $\sim 30 \mathrm{nt}$, though the length distribution can be affected by RNasel digestion conditions such as salt concentration (Ingolia et al. 2012). The undepleted libraries from this experiment have a modal fragment length of 32 , with the majority of fragments between 28 and $36 \mathrm{nt}$ in length. The libraries depleted by RiboCop and legacy Ribo-Zero have indistinguishable size distributions; importantly, however, the RNaseH-mediated depletion by the NEBNext protocol resulted in a broad distribution of fragment sizes spanning nearly the entire range of the 15 to $40 \mathrm{nt}$ size selection. These data indicate that substantial off-target RNaseH cleavage has occurred. Since we performed RNA depletion immediately after linker ligation (Fig. 1A), we surmised that this trimming was due to an abundance of leftover DNA linker, which contains six random nucleotides at its $5^{\prime}$ end that can anneal to our ribosome footprints and mediate RNAseH cleavage. To test this hypothesis, we repeated the profiling protocol, this time in K562 cells, with NEBNext depletion prior to linker ligation. We also tested the protocol with two different ranges of gel-purified fragments, 20-34 nt, which corresponds to the footprint of a single ribosome, as well as 20-60 nt, which also includes the larger footprints of collided disomes (Guydosh and Green 2014), but which typically results in more rRNA contamination. The resulting depleted libraries contained substantially fewer short and unmappable reads than those produced with depletion postligation (compare Fig. 1D to Fig. 1B; Supplemental Table S1), suggesting that many of the short and unmappable fragments in our initial experiment were the result of widespread cleavage mediated by contaminating DNA linker. These data sets reveal a much tighter size distribution than post-ligation depletion, with peaks of ribosome-protected fragments 29 and 21 nt in length (Fig. 1E), which correspond to ribosomes with occupied and unoccupied $A$ sites, respectively (Wu et al. 2019). However, there was still noticeable trimming of the ribosome footprints, particularly the $29 \mathrm{nt}$ fragments, which we presume is off-target activity from the DNA probes in the NEBNext kit.

We next tried three additional commercial rRNA depletion kits. These included a prerelease version of Ribo-Zero plus (Illumina) (a targeted depletion method similar to NEBNext that utilizes a proprietary nuclease), QIAseq
FastSelect (Qiagen) (a pool of locked nucleic acids that block reverse transcription), and riboPOOL (siTOOLs biotech) (a cocktail of biotinylated oligos against the rRNA). We performed Ribo-Zero plus before linker ligation, and QIAseq and RiboPOOL depletion immediately before reverse transcription (Fig. 1A). We performed variations of Ribo-Zero plus depletion with supplementary oligos against abundant rRNA fragments $(\mathrm{O})$, as well as supplemented with formamide (F) during probe annealing, to better denature highly structured rRNA fragments. For riboPOOL depletion, we also performed a variation where we omitted the final heating step. None of these methods proved effective at increasing the fraction of CDS-mapping fragments (Fig. 1F; Supplemental Table S1). Although Ribo-Zero plus decreased the fraction of rRNA-mapping reads, it appears that CDS-mapping reads were degraded, and the library contained an abundance of unmappable or intronic reads. These effects are evident in the CDS-mapping footprint length distribution (Fig. 1G), which shows a broadening of the 21 and 29 nt peaks and a visible increase in the fraction of 23-25 nt reads, which likely are degradation product of the longer $28 \mathrm{nt}$ fragments, that correlates with the extent of depletion. This fraction increases from $7 \%$ in the undepleted sample to $20 \%$ in the Ribo-Zero plus OF library. We interpret this result as indicating that off-target activity is a general feature of nuclease-mediated rRNA depletion.

Since the NEBNext kit was modestly successful at rRNA depletion, we tested if these depleted libraries were still suitable for making gene-level inferences of ribosome footprint density. The RPM (reads per million CDS-mapping reads) values for our undepleted or NEBNext depleted libraries were reproducible (Pearson $r^{2}=0.900$ and 0.922) between pseudoreplicates (pseudo because we isolated different fragment sizes) despite their modest sequencing depth, but many transcript RPMs differed between the depleted and undepleted libraries (Pearson $r^{2}=0.788$ and 0.780 ), with a small number of genes showing extreme deviations in RPM (Fig. 2A). These differences indicate that even aggregate gene-level measurements are perturbed by RNaseH-mediated depletion. This perturbation was also seen in the Ribo-Zero plus depleted samples (Fig. 2B), but not in samples depleted with legacy Ribo-Zero or riboCop (Fig. 2C), suggesting that it is a general feature of nuclease-mediated depletion.

An important feature of ribosome profiling data is the nucleotide-resolution positional information, which provides information on ribosome movement at specific codons or groups of codons (Ingolia et al. 2009, 2011; Stadler and Fire 2011; Zinshteyn and Gilbert 2013; Nedialkova and Leidel 2015), as well as the A site status (occupied or empty) of the ribosome, which is inferred from the length of the footprint (Wu et al. 2019). These analyses require the identification of the $A, P$, and $E$ site locations within each footprint, as well as the length class $(\sim 21,28$, etc.) of that footprint. Site identification is generally accomplished by 

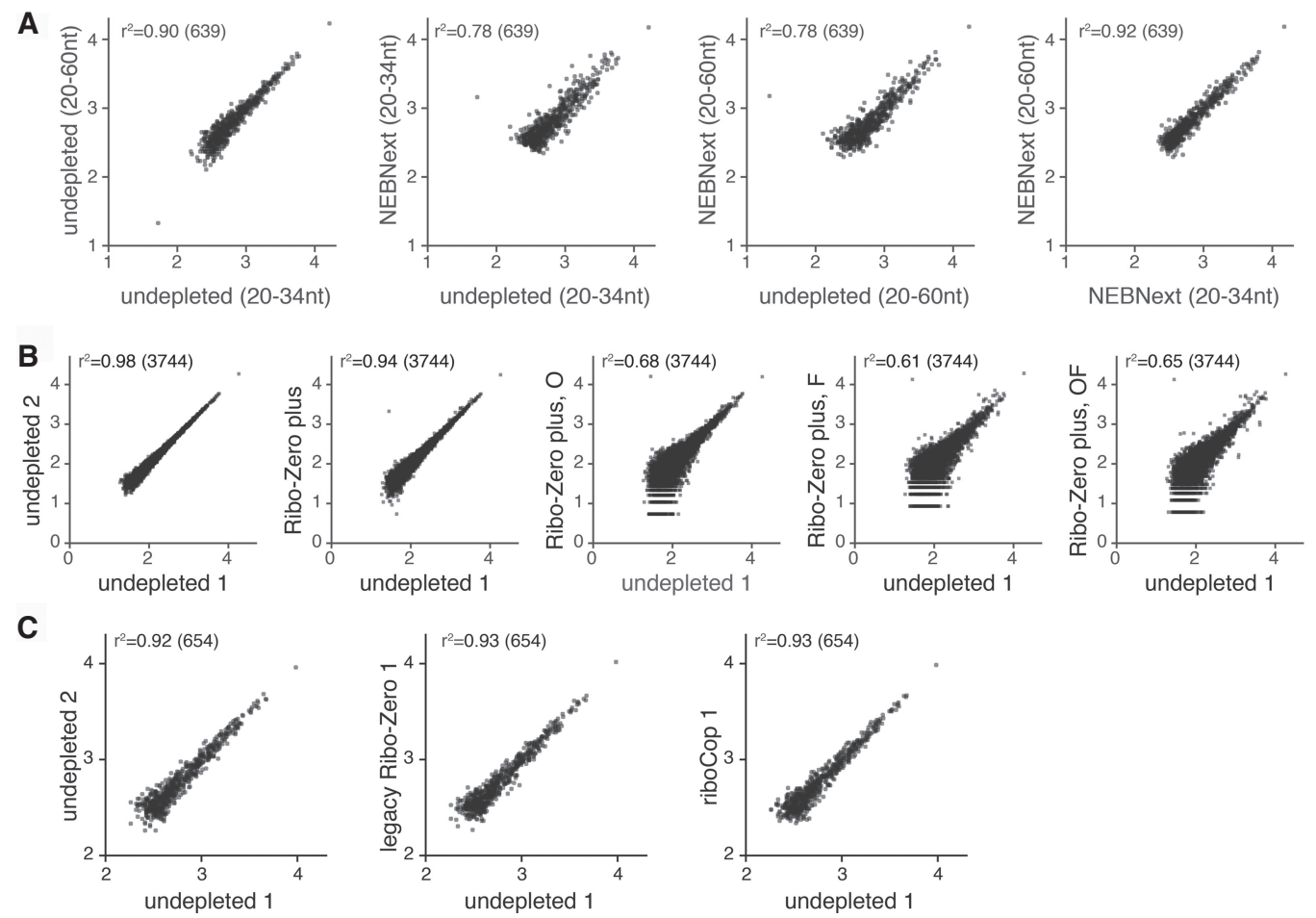

FIGURE 2. Effect of nuclease-mediated rRNA depletion on gene-level expression measurements. Correlations between log ${ }_{10}$ CDS-mapping RPM (reads per million) for replicates, and for libraries depleted with (A) NEBNext or (B) Ribo-Zero plus (C) legacy Ribo-Zero and riboCop. Pearson correlation of log-transformed values is indicated. Fragments of all lengths were included for computing RPMs for all panels.

averaging reads of a given length relative to all start codons, with the knowledge that ribosomes initiate with the start codon in the P site. Both NEBNext and Ribo-Zero plus depletion blurred the accuracy of site assignment (Fig. 3) as evidenced by reduced peaks and shallower troughs in the average gene plots.

Ribosome profiling libraries with perturbed read lengths could still be useable for positional analysis if the read trimming happens in a defined way. To see how the alterations of read lengths affect the positional resolution of ribosome profiling, we summed the total number of footprint $5^{\prime}$ ends for each read length relative to the start codons of all coding regions (Supplemental Figs. S1, S2). Ribosome profiling libraries generally have a peak of footprint $5^{\prime}$ ends mapping $\sim 12 \mathrm{nt}$ upstream of start codons. In the $5^{\prime}$-aligned footprint heatmaps in Supplemental Figures S1, S2, diagonal lines from the start codon peak are characteristic of footprints from initiating ribosomes that are shortened by nibbling at the $5^{\prime}$ end, while vertical lines indicate variation in the $3^{\prime}$ end, which maintains the position of the $5^{\prime}$ end while shortening the footprint. The presence of both vertical and diagonal lines emanating from the start codon peaks of the NEBNext-depleted libraries (Supplemental Figs. S1B, S2) indicates that the reads are trimmed from both ends. Since it is impossible to determine from which end a particular read is truncated, this trimming is unlikely to be computationally correctable.
In a final experiment, we decided to revisit previously used methods for rRNA depletion based on antisense biotinylated oligos (Ingolia et al. 2012). Based on the sequencing data from our undepleted K562 samples, we designed a set of six biotinylated oligos complementary to $37 \%$ of rRNA fragments (Supplemental Table S2). We used these oligos to subtract rRNA fragments from adaptor-ligated footprints generated from HEK293T cells. This step increased the fraction of CDS-mapping reads in our library from $5.7 \%$ to $8.7 \%$, with little or no perturbation of fragment lengths, positions, or gene-level fragment counts (Fig. 4). These promising results suggest that further efforts for ribosome profiling rRNA depletion should be aimed at optimizing custom oligo-based methods.

\section{DISCUSSION}

In summary, we have tried several rRNA depletion methods for ribosome profiling. While we do not intend this to be a comprehensive survey of the available options, we found all of the commercially available options that we tested lacking compared to legacy Ribo-Zero. For those with an immediate need some of these kits (most notably RiboCop) can be successfully used to increase usable reads for human samples, but we strongly caution against the use of any methods that use nuclease cleavage, as they blur the detailed mechanistic information that can be gained 

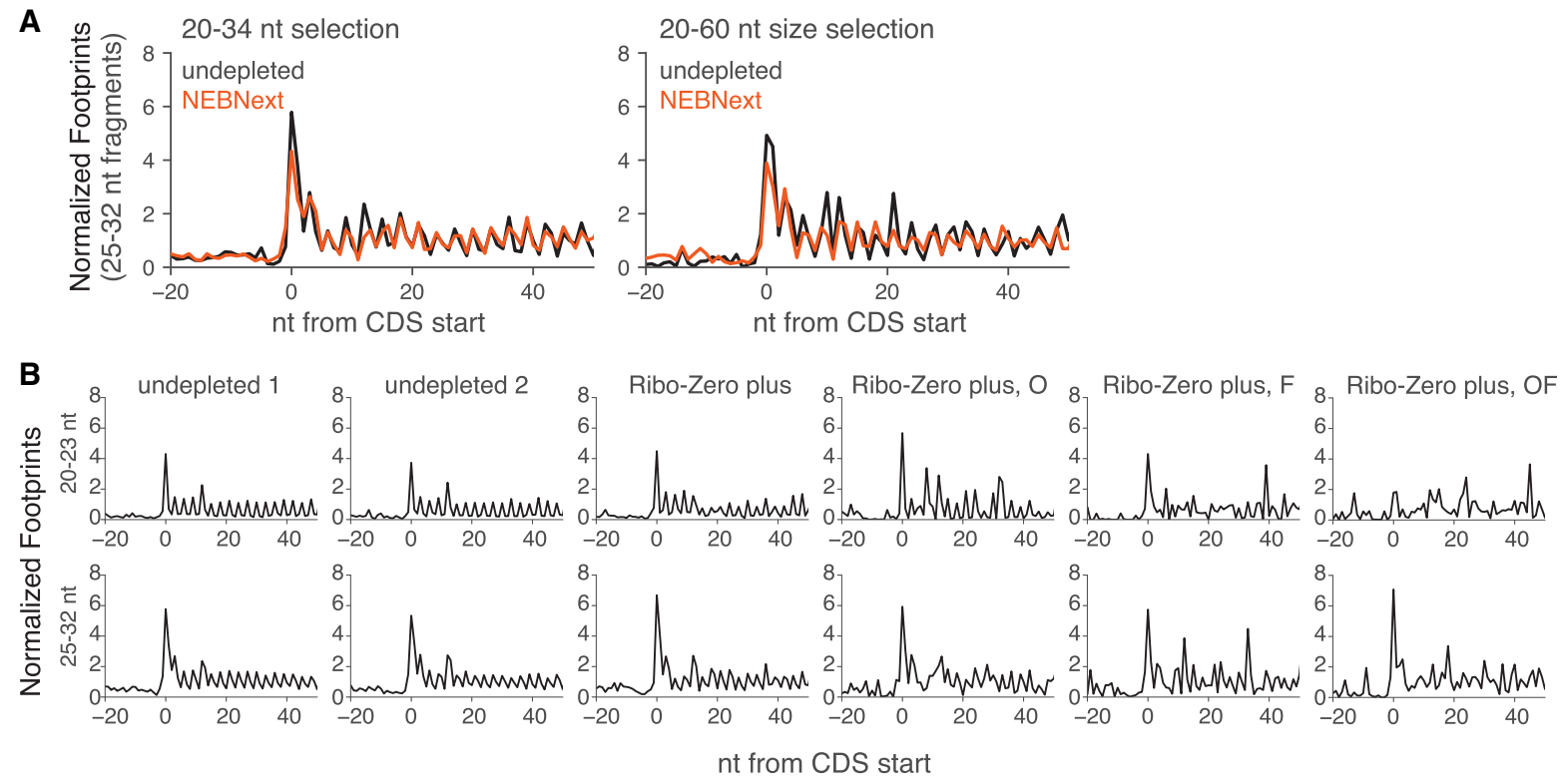

FIGURE 3. Average gene profiles for depleted data sets. Average gene plots aligned at start codons for libraries depleted with (A) NEBNext or (B) Ribo-Zero plus. Reads have been offset to indicate ribosomal $\mathrm{P}$ sites. Reads are normalized by the read density of their respective coding region, such that all genes with sufficient read numbers contribute equally, regardless of expression level. The Ribo-Zero O, F, and OF data sets have very low numbers of CDS-mapping reads, leading to low-quality average gene plots.

from the lengths of ribosome footprints (Guydosh and Green 2014; Wu et al. 2019). Moreover, most of these reagents are only available for use in a limited number of species. The best way forward for rRNA depletion in ribosome profiling may be the previously used custom oligo depletion method (Ingolia et al. 2012), which is less expensive and easier to optimize. In yeast library preparations, we have had reasonable success using previously published oligo depletion methods (Guydosh and Green 2014), consistently achieving 40\% CDS-mapping reads with libraries cut from 20-34 nt (data not shown). However, our results have been less effective for human samples (Fig. 4), indicating that further species-specific and probably sample-specific optimization in either depletion protocols or depletion oligo design is required. Fortuitously, a recently published analytical framework (Ribo-ODDR) for designing depletion oligos has the potential for substantial enrichment of CDSmapping reads (Alkan et al. 2020). Other groups have had success using double-strand specific DNase, combined with a high-temperature reannealing of the final DNA library that only leaves the most abundant sequences (the rRNA fragments) double-stranded (Chung et al. 2015), or with targeted oligos against CDNA for the most abundant rRNA contaminants (Archer et al. 2014, 2016), but these method have not to our knowledge been tested on a broad range of fragment lengths, nor with improved library preparation methods such as randomized unique molecular identifiers (McGlincy and Ingolia 2017). We note that enzymatic depletions performed at the cDNA or dsDNA stage have the particular advantage that they cannot change the ends of a cloned fragment, but only completely remove it from the sequenced pool, since removal of the ligated adaptors will prevent sequencing. Recent reports indicate that optimization of RNase digestion conditions (Sharma et al. 2019) as well as judicious choice of RNase enzyme (Gerashchenko and Gladyshev 2017) can also reduce the rRNA content of ribosome profiling libraries, though many procedures using these different nucleases obscure the high-resolution information on ribosome position and A site status. Finally, it is important to note that off-target $\mathrm{RNaseH}$ activity will not only affect the conclusions of ribosome footprint profiling experiments, but of any protocol that requires precise knowledge of the $5^{\prime}$ and $3^{\prime}$ ends of RNA fragments.

\section{MATERIALS AND METHODS}

\section{Cell culture conditions}

Mammalian cell lines were grown in cell culture incubators at $37^{\circ} \mathrm{C}$ in the presence of $5 \% \mathrm{CO}_{2}$. HEK293T cells were cultured in Dulbecco's modified Eagle medium (DMEM) $+10 \%$ fetal bovine serum (FBS) (Gibco). K562 cells were grown in Roswell Park Memorial Institute (RPMI) 1640 media + 10\% FBS.

\section{Cell lysis and ribosome footprint profiling}

Our protocol is adapted from McGlincy and Ingolia (2017). Ten centimeter dishes of adherent HEK293T cells were briefly washed with PBS and lysed by addition of $0.5 \mathrm{~mL}$ of mammalian footprint 

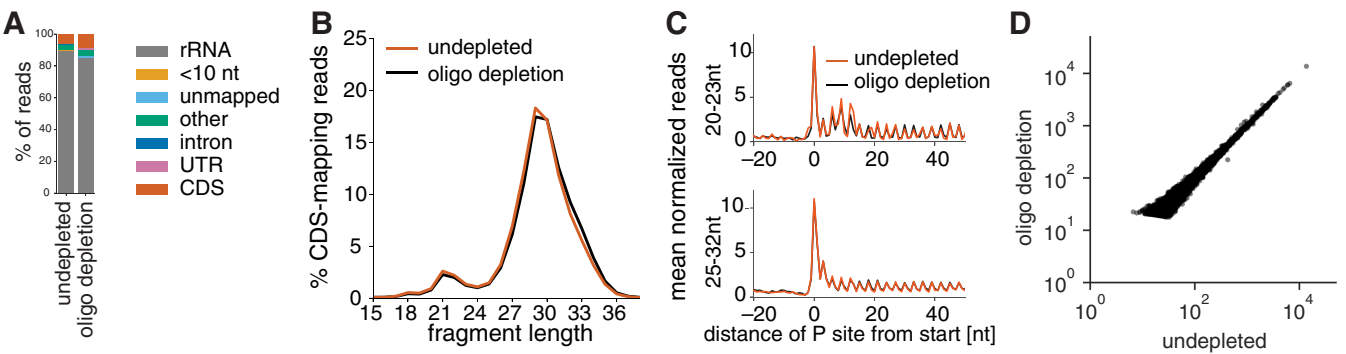

FIGURE 4. rRNA depletion with targeted biotinylated oligos. (A) Fractions of reads that are too short, unmappable, or mapping to various genomic regions for antisense oligo rRNA depletion in HEK293T cells. (B) Length distribution of ribosome footprints mapping to coding regions. (C) Average gene plots aligned at start codons for undepleted libraries or oligo depleted libraries, broken up by read length. (D) Correlations between CDS-mapping RPMs for undepleted libraries or oligo depleted libraries.

lysis buffer [20 mM Tris-Cl (pH8.0), $150 \mathrm{mM} \mathrm{KCl,} 5 \mathrm{mM} \mathrm{MgCl}$, $1 \mathrm{mM}$ DTT, $1 \%$ Triton X-100, 2 units/mL Turbo DNase (Thermo Fisher), $0.1 \mathrm{mg} / \mathrm{mL}$ cycloheximide (Sigma-Aldrich)] and vigorous scraping. 5-10 million K562 cells were harvested by centrifugation at $500 \mathrm{~g}$ for $2 \mathrm{~min}$ at $37^{\circ} \mathrm{C}$, washed briefly in $1 \mathrm{~mL} 37^{\circ} \mathrm{C}$ PBS, and lysed by pipetting in $200 \mu \mathrm{L}$ mammalian footprint lysis buffer. Lysates were incubated on ice for $10 \mathrm{~min}$ and then clarified by centrifugation at $>15,000 \mathrm{~g}$ for $10 \mathrm{~min}$. $0.5 \mathrm{~A} 260$ units of lysate was digested with 750 units of RNase I (Ambion) in $300 \mu \mathrm{L}$ lysis buffer at $25^{\circ} \mathrm{C}$ for $1 \mathrm{~h}$. Reactions were quenched with $200 \mathrm{U}$ SUPERase-in (Thermo Fisher) and pelleted over a sucrose cushion for $1 \mathrm{~h}$ at 100,000 RPM. Pellets were resuspended in TRIzol (Thermo Fisher) and RNA extracted with the Zymo Direct-zol RNA miniprep kit. Fragments were size selected on a 15\% TBEurea PAGE gel (BioRad), cutting between RNA markers of 15$40,20-34$, or 20-60, depending on the experiment. After gel elution, fragments are dephosphorylated, ligated to preadenylated 3' linker oBZ407 (AppNNNNNNCACTCGGGCACCAAGGAC/ $3 \mathrm{ddC} /$ ), reverse transcribed using protoscript II (NEB) and RT primer oBZ408 (/5Phos/RNNNAGATCGGAAGAGCGTCGTG TAGGGAAAGAGTGTAGATCTCGGTGGTCGC/iSP18/TTCAGA CGTGTGCTCTTCCGATCTGTCCTTGGTGCCCGAGTG), circularized with Circ Ligase (Lucigen), and PCR amplified. Amplified libraries were quantified by bioanalyzer high-sensitivity DNA chip (Agilent), pooled and sequenced on an Illumina HiSeq 2500 with 50 or $100 \mathrm{nt}$ reads.

\section{rRNA depletion methods overview}

For Figure 1A,B, Legacy Ribo-zero (Illumina), RiboCop (Lexogen), and NEBNext (NEB) depletions were performed after linker ligation according to the manufacturer's recommendations. For Ribo-Zero depletion, we omit the final heating step in the manufacturer's protocol, as this has been suggested to improve depletion of small fragments (McGlincy and Ingolia 2017). For subsequent figures NEBNext and Ribo-Zero plus (Illumina) depletion were performed right after fragment size selection, while all other methods were performed after linker ligation. These methods were performed according to manufacturer recommendations, except that for some experiments with Ribo-Zero Plus we included $45 \%$ formamide in the hybridization reaction (indicated as F), as well as supplementary oligos (indicated as O), provided by Illumina, designed against abundant rRNA contaminants.

\section{rRNA depletion with legacy Ribo-Zero gold kit (human/mouse/rat)}

For each sample, $100 \mu \mathrm{L}$ of Ribo-Zero magnetic beads were pelleted on a magnetic stand, washed $2 \times$ in $100 \mu \mathrm{L}$ water and resuspended in $30 \mu \mathrm{L}$ Magnetic Bead Resuspension Solution with $0.5 \mu \mathrm{L}$ RiboGuard RNase inhibitor. Two microliters of Ribo-Zero reaction buffer and $5 \mu \mathrm{L}$ Ribo-Zero Removal Solution was added to adaptor-ligated RNA in $13 \mu \mathrm{L}$ water. Solution was incubated in a thermomixer at $68^{\circ} \mathrm{C}$ for $2 \mathrm{~min}$, followed by $15 \mathrm{~min}$ at $37^{\circ} \mathrm{C}$ with 400 RPM shaking, then 5 min on ice. Full $20 \mu \mathrm{L}$ of this mixture was added to $30 \mu \mathrm{L}$ of resuspended magnetic beads, vortexed for $10 \mathrm{sec}$, and incubated for $15 \mathrm{~min}$ in a thermomixer at $25^{\circ} \mathrm{C}$. Beads were pelleted on a magnetic stand, and supernatant was kept, and isopropanol precipitated.

\section{rRNA depletion with riboCop}

Four microliters of hybridization solution and $5 \mu \mathrm{L}$ of probe mix were added to $17 \mu \mathrm{L}$ of adaptor-ligated fragments in $10 \mathrm{mM}$ tris $\mathrm{pH} 8.0$, and denatured in a thermomixer at $75^{\circ} \mathrm{C}$ for $5 \mathrm{~min}$ with shaking at $400 \mathrm{RPM}$, then annealed at $60^{\circ} \mathrm{C}$ for $30 \mathrm{~min}$ at 400 RPM. A total of $75 \mu \mathrm{L}$ of depletion beads were pelleted, washed $2 \times$ in $75 \mu \mathrm{L}$ conditioning solution, $3 \times$ in $75 \mu \mathrm{L}$ depletion solution, and resuspended in $30 \mu \mathrm{L}$ depletion solution. Annealed sample was added to beads and incubated at $60^{\circ} \mathrm{C}$ for 15 min with shaking at 400 RPM and pelleted on a magnetic bead rack for 5 min. Supernatant was kept and isopropanol precipitated.

\section{rRNA depletion with NEBNext rRNA depletion kit (human/mouse/rat)}

One microliter of NEBNext rRNA Depletion Solution and $2 \mu \mathrm{L}$ Probe Hybridization Buffer were added to $12 \mu \mathrm{L}$ size-selected fragments (or adaptor-ligated fragments for Fig. 1A) in a PCR tube and mixed by pipetting. Samples were then annealed to probes by heating to $95^{\circ} \mathrm{C}$ for $2 \mathrm{~min}$ in a thermal cycler with a heated lid set to $105^{\circ} \mathrm{C}$, cooled to $22^{\circ} \mathrm{C}$ at $0.1^{\circ} \mathrm{C} / \mathrm{sec}$ and held at $22^{\circ} \mathrm{C}$ for $5 \mathrm{~min}$. A mixture of $1 \mu \mathrm{L}$ water, $2 \mu \mathrm{L}$ RNase $\mathrm{H}$ Reaction buffer and $2 \mu \mathrm{L}$ NEBNext RNase $\mathrm{H}$ was added to the annealed mixture and mixed by pipetting. The mixture was incubated in thermal cycler with heated lid at $37^{\circ} \mathrm{C}$ for $30 \mathrm{~min}$, then immediately placed on ice. To this mixture $30 \mu \mathrm{L}$ of DNase I 
digestion master mix $(22.5 \mu \mathrm{L}$ water, $5 \mu \mathrm{L}$ DNase I reaction Buffer, $2.5 \mu \mathrm{L}$ DNase I) was added and incubated at $37^{\circ} \mathrm{C}$ for $30 \mathrm{~min}$, followed by isopropanol precipitation.

\section{rRNA depletion with Ribo-Zero plus reagent (human/ mouse/rat)}

To $3.15 \mu \mathrm{L}$ of gel purified RNA fragments, we added the following: $4 \mu \mathrm{L}$ of premixed ( $3 \mu \mathrm{L} \mathrm{DB} 1+1 \mu \mathrm{DP} 1), 1 \mu \mathrm{L}$ supplementary probes when indicated, $6.75 \mu \mathrm{L}$ formamide when indicated, water to $15 \mu \mathrm{L}$. Samples were then annealed to probes by heating to $95^{\circ} \mathrm{C}$ for $2 \mathrm{~min}$ in a thermal cycler with a heated lid set to $105^{\circ} \mathrm{C}$, and cooled to $37^{\circ} \mathrm{C}$ at $0.1^{\circ} \mathrm{C} / \mathrm{sec}$. Annealed samples were spun down and $5 \mu \mathrm{L}$ of ( $1 \mu \mathrm{L} \mathrm{RDE}+1 \mu \mathrm{L} \mathrm{RDB})$ was added, mixed, and incubated in a thermal cycler at $37^{\circ} \mathrm{C}$ for $15 \mathrm{~min}$ to digest RNA. Samples were spun down and $10 \mu \mathrm{L}$ of $(3 \mu \mathrm{L}$ PRE + $7 \mu \mathrm{L}$ PRB) was added, mixed, and incubated in a thermal cycler at $37^{\circ} \mathrm{C}$ for $15 \mathrm{~min}$ to digest DNA, and placed on ice, followed by isopropanol precipitation.

\section{rRNA depletion with OIAseq FastSelect}

Pellets of adaptor-ligated RNA were resuspended in $(10 \mu \mathrm{L}$ water + $4 \mu \mathrm{L}$ ProtoScript II buffer $+1 \mu \mathrm{L}$ Qiagen fastselect [THS-001Z-24]), transferred to a PCR strip tube, and annealed in a thermal cycler with a heated lid for 2 min each at $75^{\circ} \mathrm{C}, 70^{\circ} \mathrm{C}, 65^{\circ} \mathrm{C}, 60^{\circ} \mathrm{C}$, $55^{\circ} \mathrm{C}, 37^{\circ} \mathrm{C}, 25^{\circ} \mathrm{C}, 4^{\circ} \mathrm{C}$. Reverse transcription was then performed as usual.

\section{rRNA depletion with riboPOOL (protocol_v1-5)}

Lyophilized riboPOOL (human) was resuspended to $100 \mu \mathrm{M}$. Pellets of adaptor-ligated RNA were resuspended in (14 $\mu \mathrm{L}$ water $+5 \mu \mathrm{L}$ hybridization buffer $[10 \mathrm{mM}$ Tris- $\mathrm{HCl} \mathrm{pH} 7.5,1 \mathrm{mM}$ EDTA, $2 \mathrm{M} \mathrm{NaCl}]+1 \mu \mathrm{L}$ riboPOOL, $1 \mu \mathrm{L}$ superasin RNase inhibitor [Thermo]). The mixture was heated to $68^{\circ} \mathrm{C}$ in thermomixer for $10 \mathrm{~min}$, and the thermomixer was set to $37^{\circ} \mathrm{C}$ and allowed to slowly cool, allowing probe annealing over $30 \mathrm{~min}$. Washed 80 $\mu \mathrm{L}$ of Dynabeads MyOne Streptavidin C1, 0.1 M NaOH 0.05 M $\mathrm{NaCl}$ (Thermo Fisher \#65001) $2 \times$ in $100 \mu \mathrm{L}(0.1 \mathrm{M} \mathrm{NaOH} 0.05 \mathrm{M}$ $\mathrm{NaCl})$, then $1 \times$ in $(0.1 \mathrm{M} \mathrm{NaCl})$, then resuspended in $160 \mu \mathrm{L}$ (5 mM Tris- $\mathrm{HCl}$ [pH 7.5] 0.5 mM EDTA $1 \mathrm{M} \mathrm{NaCl}$ ) and split into two tubes. Annealed samples were added to first aliquot of beads and incubated at $37^{\circ} \mathrm{C}$ for $15 \mathrm{~min}$, followed by a $50^{\circ} \mathrm{C}$ incubation for $5 \mathrm{~min}$ (this heating was omitted where indicated). Both bead aliquots were pelleted, supernatant from second aliquot was removed and replaced with supernatant from first aliquot. Beads were incubated once more at $37^{\circ} \mathrm{C}$ for $15 \mathrm{~min}$, followed by a $50^{\circ} \mathrm{C}$ incubation for $5 \mathrm{~min}$ (omitted where indicated). Beads were pelleted and supernatant transferred to a new tube twice.

\section{rRNA depletion with custom biotinylated oligos}

To design depletion oligos, we visualized the location of the most abundant contaminating rRNA in the UCSC Genome Browser and ordered $60 \mathrm{nt} 5^{\prime}$ biotinylated oligos antisense to the most abundant fragments. An oligo mix solution was made by diluting the six depletion oligos (Supplemental Table S2) in 4x SSC buffer to a concentration of $2.5 \mu \mathrm{M}$ for each oligo. A total of $10 \mu \mathrm{L}$ of the oligo mix solution was added to $10 \mu \mathrm{L}$ of sample solution (ligation products precipitated and resuspended in nuclease-free water). The sample and oligo mixture were heated at $80^{\circ} \mathrm{C}$ for $2 \mathrm{~min}$ in a thermomixer, which was then set to $25^{\circ} \mathrm{C}$ to gradually cool the mixture and anneal oligos to the sample RNA. During cooling, $150 \mu \mathrm{L}$ of MyOne Streptavidin C1 dynabeads (ThermoFisher) per depletion were washed thrice with $150 \mu \mathrm{L}$ of $1 \times$ Binding/Washing buffer (5 mM Tris, $\mathrm{pH} 8.0,1 \mathrm{M} \mathrm{NaCl}$, $0.5 \mathrm{mM}$ EDTA), and then beads were resuspended in $30 \mu \mathrm{L} 2 \times$ Binding/Washing buffer. The cooled sample was added to the prepared beads and incubated at $25^{\circ} \mathrm{C}$ for 15 min with shaking at $500 \mathrm{rpm}$ in a thermomixer. After incubation, beads were precipitated with a magnetic stand, and supernatant was removed, and isopropanol precipitated to recover RNA.

\section{Data analysis}

Raw ribosome footprint reads were trimmed of the four random $\mathrm{nt}$ from the $5^{\prime}$ end with seqtk (https://github.com//h3/seqtk), then trimmed of $3^{\prime}$ adaptor sequence (NNNNNNCACTCGGGCAC CAAGGAC) using Skewer (Jiang et al. 2014). Reads longer than 10 nt were aligned to PhiX-174, human rRNA and human noncoding RNA sequences using STAR (Dobin et al. 2013) with -outFilterMismatchNmax 2 -outSAMmultNmax 1. Unmapped reads were mapped to the hg38 human genome (GENCODE release 27), using STAR with parameters -alignSJDBoverhangMin 1 -alignIntronMax 1000000 -alignSJoverhangMin 3 -outFilterType BySJout -outFilterMultimapNmax 200, -outFilterScoreMin OverLread 0 -outFilterMatchNminOverLread 0 -outFilterMatch Nmin 0, -outFilterMismatchNmax 3. To generate a reference transcriptome with a single transcript per gene for alignment, the GENCODE v27 gtf file was filtered for those transcripts with an APPRIS score (Rodriguez et al. 2013) between 1 and 4 (inclusive) and then the transcript with the longest CDS (and longest transcript if tied) was chosen. For multiply mapping reads, the primary alignment was used for all analyses. For Figure $1 A, C, E$, reads were assigned the smallest feature that they overlapped based on the comprehensive GENCODE v27 annotation gtf file. For RPM correlation plots, only genes with an average of 64 or more counts and a minimum of 1 RPM between all libraries in the same panel were included. The Python code for the analysis pipeline and for figure generation are available at https://github.com/borisz264/ rRNA_depletion_2020. Sequencing data have been deposited in GEO with accession GSE147324.

\section{SUPPLEMENTAL MATERIAL}

Supplemental material is available for this article.

\section{ACKNOWLEDGMENTS}

We thank David Mohr and the Johns Hopkins Genetic Resources Core Facility for sequencing assistance. We thank Scott Kuersten (Illumina) for providing Ribo-Zero Plus material as well as advice on its use, and Sezen Meydan for suggestions on the use of Qiagen FastSelect. This work was funded by the National Institutes of Health, National Institute of General Medical 
Sciences (2R37GM059425-14 to R.G.; 5K99GM135450-02 to B.Z.), Howard Hughes Medical Institute (HHMI) (R.G.), and the Cystic Fibrosis Foundation (GREEN16G0). B.Z. was an HHMI fellow of the Damon Runyon Cancer Research Foundation (DRG2250-16) for a portion of this study.

Received March 30, 2020; accepted June 1, 2020.

\section{REFERENCES}

Alkan F, Silva J, Barberà EP, Faller WJ. 2020. Ribo-ODDR: ribo-seq focused oligo design pipeline for experiment-specific depletion of ribosomal RNAs. bioRxiv doi:10.1101/2020.01.12.900175

Archer SK, Shirokikh NE, Preiss T. 2014. Selective and flexible depletion of problematic sequences from RNA-seq libraries at the cDNA stage. BMC Genomics 15: 401. doi:10.1186/1471-2164-15-401

Archer SK, Shirokikh NE, Beilharz TH, Preiss T. 2016. Dynamics of ribosome scanning and recycling revealed by translation complex profiling. Nature 535: 570-574. doi:10.1038/nature18647

Arribere JA, Fire AZ. 2018. Nonsense mRNA suppression via nonstop decay. Elife 7: e33292. doi:10.7554/eLife.33292

Brar GA, Yassour M, Friedman N, Regev A, Ingolia NT, Weissman JS. 2012. High-resolution view of the yeast meiotic program revealed by ribosome profiling. Science 335: 552-557. doi:10.1126/sci ence.1215110

Chung BY, Hardcastle TJ, Jones JD, Irigoyen N, Firth AE, Baulcombe DC, Brierley I. 2015. The use of duplex-specific nuclease in ribosome profiling and a user-friendly software package for Ribo-seq data analysis. RNA 21: 1731-1745. doi:10.1261/rna.052548.115

Dobin A, Davis CA, Schlesinger F, Drenkow J, Zaleski C, Jha S, Batut P, Chaisson M, Gingeras TR. 2013. STAR: ultrafast universal RNA-seq aligner. Bioinformatics 29: 15-21. doi:10.1093/bioinformatics/ bts635

D'Orazio KN, Wu CC-C, Sinha N, Loll-Krippleber R, Brown GW, Green R. 2019. The endonuclease Cue2 cleaves mRNAs at stalled ribosomes during No Go Decay. Elife 8: e49117. doi:10.7554/ elife.49117

Gerashchenko MV, Gladyshev VN. 2017. Ribonuclease selection for ribosome profiling. Nucleic Acids Res 45: e6. doi:10.1093/nar/ gkw822

Guo H, Ingolia NT, Weissman JS, Bartel DP. 2010. Mammalian microRNAs predominantly act to decrease target mRNA levels. Nature 466: 835-840. doi:10.1038/nature09267

Guydosh NR, Green R. 2014. Dom34 rescues ribosomes in 3' untranslated regions. Cell 156: 950-962. doi:10.1016/j.cell.2014.02.006

Ingolia NT, Ghaemmaghami S, Newman JRS, Weissman JS. 2009. Genome-wide analysis in vivo of translation with nucleotide resolution using ribosome profiling. Science 324: 218-223. doi:10 $.1126 /$ science. 1168978

Ingolia NT, Lareau LF, Weissman JS. 2011. Ribosome profiling of mouse embryonic stem cells reveals the complexity and dynamics of mammalian proteomes. Cell 147: 789-802. doi:10.1016/j.cell .2011 .10 .002

Ingolia NT, Brar GA, Rouskin S, McGeachy AM, Weissman JS. 2012. The ribosome profiling strategy for monitoring translation in vivo by deep sequencing of ribosome-protected mRNA fragments. Nat Protoc 7: 1534-1550. doi:10.1038/nprot.2012.086

Ingolia NT, Hussmann JA, Weissman JS. 2019. Ribosome profiling: global views of translation. Cold Spring Harb Perspect Biol 11: a032698. doi:10.1101/cshperspect.a032698

Jiang H, Lei R, Ding S-W, Zhu S. 2014. Skewer: a fast and accurate adapter trimmer for next-generation sequencing paired-end reads. BMC Bioinformatics 15: 182. doi:10.1186/1471-2105-15182

Kozak M, Shatkin AJ. 1976. Characterization of ribosome-protected fragments from reovirus messenger RNA. J Biol Chem 251: 4259-4266.

Lareau LF, Hite DH, Hogan GJ, Brown PO. 2014. Distinct stages of the translation elongation cycle revealed by sequencing ribosomeprotected mRNA fragments. Elife 3: e01257. doi:10.7554/eLife .01257

McGlincy NJ, Ingolia NT. 2017. Transcriptome-wide measurement of translation by ribosome profiling. Methods 126: 112-129. doi:10 .1016/j.ymeth.2017.05.028

Nedialkova DD, Leidel SA. 2015. Optimization of codon translation rates via tRNA modifications maintains proteome integrity. Cell 161: 1606-1618. doi:10.1016/j.cell.2015.05.022

Rodriguez JM, Maietta P, Ezkurdia I, Pietrelli A, Wesselink J-J, Lopez G, Valencia A, Tress ML. 2013. APPRIS: annotation of principal and alternative splice isoforms. Nucleic Acids Res 41: D110D117. doi:10.1093/nar/gks1058

Sharma P, Nilges BS, Wu J, Leidel SA. 2019. The translation inhibitor cycloheximide affects ribosome profiling data in a species-specific manner. bioRxiv doi:10.1101/746255

Sooknanan RR. 2009. Provisional patent application no. 61/234,044; filed on August 14, 2009.

Stadler M, Fire A. 2011. Wobble base-pairing slows in vivo translation elongation in metazoans. RNA 17: 2063-2073. doi:10.1261/rna .02890211

Steitz JA. 1969. Polypeptide chain initiation: nucleotide sequences of the three ribosomal binding sites in bacteriophage R17 RNA. Nature 224: 957-964. doi:10.1038/224957a0

Wolin SL, Walter P. 1988. Ribosome pausing and stacking during translation of a eukaryotic mRNA. EMBO J 7: 3559-3569. doi:10 .1002/j.1460-2075.1988.tb03233.x

Wu CC-C, Zinshteyn B, Wehner KA, Green R. 2019. High-resolution ribosome profiling defines discrete ribosome elongation states and translational regulation during cellular stress. Mol Cell 73: 959-970.e5. doi:10.1016/j.molcel.2018.12.009

Zinshteyn B, Gilbert WV. 2013. Loss of a conserved tRNA anticodon modification perturbs cellular signaling. PLoS Genet 9: e1003675. doi:10.1371/journal.pgen.1003675 

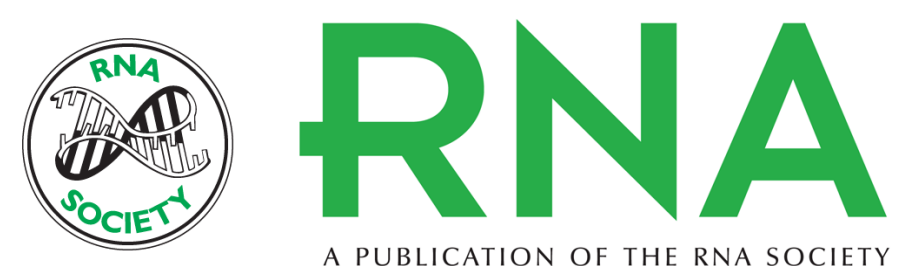

A PUBLICATION OF THE RNA SOCIETY

\section{Nuclease-mediated depletion biases in ribosome footprint profiling libraries}

Boris Zinshteyn, Jamie R. Wangen, Boyang Hua, et al.

RNA 2020 26: 1481-1488 originally published online June 5, 2020

Access the most recent version at doi:10.1261/rna.075523.120

\section{Supplemental http://rnajournal.cshlp.org/content/suppl/2020/06/05/rna.075523.120.DC1 \\ Material}

References This article cites 27 articles, 8 of which can be accessed free at: http://rnajournal.cshlp.org/content/26/10/1481.full.html\#ref-list-1

Creative This article is distributed exclusively by the RNA Society for the first 12 months after the Commons full-issue publication date (see http://rnajournal.cshlp.org/site/misc/terms.xhtml). After 12

License months, it is available under a Creative Commons License (Attribution-NonCommercial 4.0 International), as described at http://creativecommons.org/licenses/by-nc/4.0/.

Email Alerting
Service

Receive free email alerts when new articles cite this article - sign up in the box at the top right corner of the article or click here.

To subscribe to $R N A$ go to:

http://rnajournal.cshlp.org/subscriptions 